\section{Knowledge Society and Rising Imperatives of Sustainable Energy Technology: Socio-Cultural and Economic Dimensions}

\section{Harald E. Otto}

\begin{abstract}
Department of Industrial Engineering and Mathematical Sciences, Polytechnic University of Marche, Ancona I-6o131, Italy
\end{abstract}

Received: January 24, 2014/ Accepted: February 3, 2014

\begin{abstract}
In addition to technological challenges, sustainable energy systems face a host of difficulties related to their deployment, diffusion, and use, and at the same time they create an impact on the environment and society. Within a broader systemic socio-economic and technological viewpoint, a stance which is widespread and acceptable nowadays is to regard the development of technology as socially-culturally and economically conditioned, though not determined, as was claimed by many scholars in the past. On the other hand, as artifacts and technology in a wider sense do not create, and change themselves, and do not even autonomously determine themselves, they represent without doubt important factors that impact social-cultural and economic change, depending not so much on their existence per se, but on the way in which they are deployed and used. In the work presented in this paper, given the context and viewpoint as outlined, two directions are considered, namely the deployment and diffusion of renewable energy systems and behavior change in energy use. Both are considered within the context of developed industrial nations. Efforts aimed at shedding some light on their conditioning factors and possible interrelationships are approached from a multi-disciplinary and interdisciplinary view taking into account insight and empirical results from various fields of study. These are analyzed and related to the characteristics of rising knowledge societies, the new role of knowledge generation, and knowledge itself in respect to various types of innovation, and also the new dynamics of rising knowledge economies.

Keywords: renewable energy systems; energy efficiency; sustainable development; knowledge society; knowledge economy
\end{abstract}

* Corresponding AuthorrTel.: +39-071-220-4800; fax: +39-071-2204801.; E-mail address: h.e.otto@univpm.it

\section{Introduction}

In the face of efforts to address issues of climate change related to the mitigation of greenhouse gas emissions and rapidly declining resources of fossil fuels, the development and deployment of sustainable energy systems is facing many challenges. These will eventually require drastic changes affecting many, if not all, social, legislative, and economic actors, as well as the structures of public and private organizations down to individual households. Seen from the more holistic viewpoint of technical and socioeconomic systems, this in turn requires changes in technology. Social change and technological change are interrelated through forces and factors pertaining to technical, social, cultural, political and legal operating directions. Which technologies and which particular artifacts are adopted, on what scale and at what speed of diffusion, depends not only on their properties and defining qualities but also on the characteristics of society, which has its own environment of deployment, in addition to the natural and built environment. On the other hand, in the socio-historical and technological-historical context technology represents an important factor not only in facilitating social-cultural and economic organizations and structures, but also in their change.

In this paper, within the context and issues as outlined above, two directions are considered, namely the deployment and diffusion of renewable energy systems and behavior change in energy use. Both are examined within the context of developed industrial nations. Efforts aimed at shedding some light on their conditioning factors and possible interrelationships are approached from a multidisciplinary and interdisciplinary view taking into account insights and empirical results from various fields of study. These are then analyzed and related to the characteristics of rising knowledge societies, the new role of knowledge generation and knowledge itself in regard to various types of innovation, and also the new dynamics of rising knowledge economies.

\section{Post-Modern Dynamics of Society, Knowledge, and Economy}

The rise of new knowledge economies in developed nations has changed not only the economic dynamics and values, but also the nature of society. Knowledge has become the main resource in respect to products, services, processes, and business models. This is in contrast to traditional industries, where land, capital, and labor were largely relevant. Knowledge has different properties from traditional commodities. Knowledge does not diminish by use (non-rivalry). It is difficult to prevent others from using it (partial excludability). It is a kind of input and output with current innovations being the input of the next set of 
innovations. Knowledge also has positive externalities such as spillovers. For example, investment in research and education benefits many. There is also positive feedback reflected in increasing returns. For example, in relation to utilization and supply, the more you use and know the easier it is to use and acquire.

With these new economic values and dynamics, social relations, culture, and institutions are undergoing change, resulting in what is now commonly termed the knowledge society. There are now several different views regarding the nature of currently developing knowledge societies and relating to the primacy of scientific knowledge, the rise of knowledge work, and the increasing interconnection of work and social relations enabled by information and communication technology. Within such a scenario, obviously an open culture is not only encouraged, but also technologically supported. In this culture, information is made publicly available. Unmediated communication and collective thinking are developed. Social mobilization occurs, and there is decentralized production, distribution, and consumption of knowledge (cf. $[1,2,3]$ ).

\section{Knowledge and Diffusion of Technology}

The deployment of new and complex technology systems, such as renewable energy systems, and subsequent efforts to integrate them into the social environment, requires the acceptance of various social actors. To achieve effective as well as efficient results regarding the diffusion of a particular technology, acceptance needs to be negotiated and, based on the out come, modifications and adjustments need to be carried out resulting in change affecting both the technology and its way of deployment and use, and the social environment. This requires considerable knowledge about and insight on how all the aspects of technological and social change are interrelated, especially if innovation is to be used as a means to achieve progress. Since innovation relates to all cycles of a technology from design and manufacturing to deployment and use, the latter can be an important opportunity and source of learning (cf. [4,5]), thus contributing to innovation itself. However, to translate this potential into any practical benefit, both the activities and processes of knowledge generation and the knowledge created, as related to each cycle, need to be linked and coordinated.

\subsection{The social dimension}

Unfortunately, in relation to deployment and use, especially in the case of renewable energy systems, everything lags far behind. This is partly due to our still limited understanding of the inner workings and structures of current sociotechnical systems. However, it is also related to the fact that, as a consequence of this lack, studies in complex sociotechnical systems have not yet achieved what has already been common practice for quite some time now in natural and engineering science knowledge generation. What is needed is to have, in a Kuhnian sense [6], an accepted paradigm that facilitates so-called normal research, i.e. that somewhat guides and coordinates knowledge generation based on theory forming, experimental research and empirical evidence, which in turn provide the input necessary for further innovation and development.

One promising approach which seems worth pursuing represents efforts to relate insights from different fields of studies to both the social dimension of renewable energy system diffusion and knowledge creation through learning within technology deployment and use. These different fields of studies include the social shaping of technology, science and technology studies, technology assessment, and social acceptance studies. In the following, an attempt is made in this sub-section to outline the basics related to the social dimension, while considerations related to the cultural and economic dimension are discussed in the following two sub-sections, though a detailed in-depth study would go far beyond the scope of this paper.

To begin with, it is helpful to regard a complex technological system as a configuration $[4,7]$ which is customized to fit requirements stemming from structures, processes, and actors of a particular environment subject to deployment. In this context, adjustments are not limited to the technical aspects and artifacts of a technology, but include also the interface to the social environment, i.e. aspects of use and benefit distribution in respect to where, when, how and by whom. Efforts to overcome an initial misfit between a complex technological system and its deployment environment already represent innovation and create knowledge by means of so-called learning by trying, also termed innofusion [8]. A typical example is the efforts of energy developers or utilities to negotiate the deployment of wind farms or solar photovoltaic installations with various social actors such as local authorities and community members.

Here it is important firstly to gain knowledge on factors of misfit regarding siting decisions for renewable energy projects related to interpretations of backyard-protective reactions such as place attachment $[9,10]$ and spatial proximity issues [11], and aspects of securing and distributing fairly benefits for the community $[12,13]$ and looking further into their possible cause. Then, however, it is also necessary to document and integrate our understanding and insight stemming from actual individual 
cases on how solutions have been achieved and to make this information available as a contribution to the cumulative knowledge on renewable technology systems. This is especially important in the context of renewable energy system deployment. In this field, innovation with input from knowledge generated through learning by use is limited later in the use phase, because possibilities of adjustment after deployment are limited. This is in stark contrast to the possibilities with other complex technologies, such as integrated information technology systems, for example. Here knowledge generation based on what is referred to as user innovation [14] has brought a considerable contribution to the success of the development and diffusion of information technology. Of course, one essential difference, which also makes the application of user innovation less practicable in the context of renewable energy systems, is the fact that in the case of the information technology systems, the user, whether it be an individual end user / household or a company, is both the actor who is conceiving, proposing and even executing changes and modifications to the system and the recipient of the benefits following thereafter. In the case of renewable energy systems, circumstances are different, because actual users and beneficiaries are usually different stakeholders and actors such as energy developers, utilities, investors, and local community members, each with a different viewpoint and goal set.

\subsection{The cultural dimension}

Yet another dimension equally important, but often overlooked and neglected, is defined by cultural characteristics. This dimension can be found within the defining quality of many characteristics of social structures and social actors, and in the way certain processes are structured and executed. This is commonly reflected in what is known as institutional culture, educational culture, corporate culture, market culture, etc. It is also an element related to the forming of beliefs, opinions, and how we evaluate and reach decisions. In the context of the last, besides information and knowledge, cultural factors play an important role in risk perception (cf. [15,16]).

Unfortunately, empirical cross-cultural studies on energy technology preferences and public and social acceptance of renewable energy systems related to issues of the cultural dimension are almost non-existent. The increasing importance of this issue was pointed out by a very recent empirical study [17], which was aimed at further insight into the opinion-based public preferences towards electricity technologies and future low-carbon portfolios. The study showed considerable differences in stated preferences, and these were attributed to some degree to socio-demographic variables, such as age and level of education, and a strong country/culture bias towards particular technologies and portfolios. However, it is important here to note that we should not make the somewhat simplistic mistake of assuming that public acceptance is equal to social acceptance. The reduction of social acceptance to public attitudes and opinions, which are related to the level of the individual, namely the ordinary citizen, ignores the position and perspectives of all the other social actors ranging from public organizations to companies, which, in many cases, have a much stronger influence on the decision and implementation processes (see also discussions in $[18,19]$ ).

The study also provided evidence on the important roles of communication and information provision. They represent crucial components in promoting public support in concert with efforts to integrate citizens and stakeholders into political decision-making and the forming of public policies aimed at increasing the transparency and legitimacy of governance processes. Not only are these issues important in their own right, but they gain significant momentum when considered now with the post-modern knowledge societies which are arising in different geographical and cultural regions, as discussed further elsewhere in this paper.

\subsection{The economic dimension}

To successfully deploy innovative renewable energy technology within a wider socio-technological system, several conditions need to be considered. These conditions are related to market acceptance, also sometimes known in social acceptance studies as a process of market adoption. In addition, in the case of complex technologies such as energy systems, dependencies on certain infrastructures, such as the power grid, and access to those, impose additional requirements on the deployment and diffusion. There have been some quite successful diffusion approaches involving innovative artifacts and technologies in information technology and biotechnology, and there have been studies of those approaches. As is already known from such earlier studies, to accomplish a certain commercial potential that can actually be translated into a successful market acceptance, it is necessary to create customers and a market where there were few or none before. Also, innovation and development are not just about deploying technological artifacts and know-how, but involve other types of knowledge and expertise as well (cf. [20]). In view of the urgency of mitigation efforts related to greenhouse gas emissions and climate change, the situation regarding renewable energy system diffusion is somewhat different from that of technology diffusion in the case of information technology and biotechnology. Here the question is to what 
extent governments should take an active role by means of policy instruments in the support and regulation of levels of speed and scales of deployment.

Energy policy mechanisms currently applied in most countries can be sub-divided into two basic types of instruments, namely quantity-based and price-based. The former are instruments such as renewable portfolio standards strongly supported by industry and utilities. The latter are auctions and feed-in tariffs, also referred to as premium payments or minimum price standards, which support a more diverse participation of social actors, thus being also more attractive for decentralized small-scale and community-based projects. Feed-in tariffs are so-called demand-pull instruments [21,22], due to government efforts to create markets for renewable energy technologies. They are currently applied in at least 61 countries, though over half of these measures have been enacted only since 2005 [23]. Feed-in tariffs are often seen as effective policy instruments, due to their capacity to remove market barriers, thus enabling a rapid deployment of significant capacity (for a good overview see [24]). This is also considered an important factor for innovation, as new and novel technologies usually remain too costly compared to their conventional counterparts. Feed-in tariffs help the new technologies to make a swift and timely transition from the niche markets they reside in into the general market [25]. Now, if innovation and development accelerate and a new technology becomes less expensive in terms of production and deployment costs, support of feed-in tariffs should recede and make way for a more natural market competition. However, due to the structure of feed-in tariffs in the first place, which is aimed at providing stability and reduced uncertainty and risk for investors, they paradoxically counter-act their own purpose over time in the case of success, eventually resulting in cost escalation and thus also undermining public support. An example is the recent revision of feed-in tariffs for solar photovoltaic installations around the world, resulting in tariff cuts of up to $45 \%$ in many developed European countries. This can be seen as a countermeasure to dampen the booming rate of installations, and was due to an unprecedented steep decline in prices of solar photovoltaic technology that took place mostly in 2009-2010 [23].

Taking a second look at such a scenario, it seems that there is another option besides market creation. This second option is to directly further innovation and development, and this also seems a viable choice for subsidies-based support. This is a position that has been argued for by advocates of the so-called technology-push approach (cf. [26]), which stipulates that governments should allocate funding for the innovation of renewable energy systems driven by knowledge and know-how generation and research and development. For modern industrialized nations, this approach is indeed a viable option. However, considering the characteristics of rising knowledge societies and concomitant phenomena attributable to the likewise rising knowledge economies, several newly developing issues, perhaps manifesting themselves as new barriers apparently inhibiting progress, need to be taken into account.

There are current developments to secure copyright protection for the digital computerized reproduction and distribution of information and there are growing efforts to assert and enforce intellectual property rights over technological knowledge, mostly through the use of copyrights, patents and other novel instruments of legal protection. Because of these moves, the availability of and access to knowledge are about to be severely restricted at the expense of public domain knowledge. This is a typical characteristic of socio-technological systems, where economic factors and commercial interests weight in during rapid deployment of a new technology, which creates scarcity where there was none before. In this case, it creates an artificial scarcity of technological knowledge and knowhow. This effect combines unfortunately with the translation of knowledge from a public good into a commodity, though the characteristics and mechanisms of production, improvement, and distribution/diffusion of knowledge and actual (tangible) commodities are in most parts incommensurable (see discussions in $[27,28,29]$ ). This situation may lead to underinvestment in innovation of renewable energy technology, because commercial and industrial actors fear that they will be unable to fully capture and exploit the benefits from their innovation efforts. Some portion of this benefit will be shifted to other social and market actors, even though knowledge seems to possess the natural characteristics of a public good. This tendency has received considerable attention in postmodern economics literature, and is often considered to be an innovation market failure. Another aspect associated with innovation market failure is the spillover effects of knowledge and technological know-how. This is especially the case with the latter, which significantly improves through what is called learning-by-doing, referring to empirical observations that the costs of production tend to decline as the cumulative production of new technologies increases. In a similar fashion, collective knowledge (as a public good, not a commodity) quickly improves and develops, thus furthering innovation and new discoveries, if it is used, exchanged, and commented upon by many social actors simultaneously. This is made possible due to its characteristics of being non-rival in use, and having low 
excludability combined with multiplicative effects referred to as the shoulders of giants effect. However, since policymaking is a complex and difficult process, which is greatly influenced by powerful interest groups, usually lobbying for particular policy designs and price systems, outcomes regarding the efficiency and effectiveness of actual implementations of policy instruments may vary considerably from theory and projections.

\section{Knowledge and Behavior Change}

This section represents the second part of a broader view of sustainable energy technology, and it considers, in addition to the technology itself, the importance of choices regarding its deployment and use, issues related to knowledge deficiency and knowledge generation in respect to innovation, and how these relate to the characteristics of rising knowledge societies. Although, as proved by many empirical studies, the potential here for improving energy efficiency is considerable, there still seem to be many barriers to successfully translating what we know from insight provided by behavioral and social science, and by environmental psychology, into behaviorally informed energy conservation programs on a larger scale.

\subsection{The social dimension}

As consistently shown by empirical research, there are three basic factors which influence energy conservation most. Those three are an awareness of needs, knowledge of ways to achieve those needs, and an environment that enables the adoption of relevant behavior. Although private and public organizations, as well as the general public per se, are generally aware of the problems related to energy use and issues of greenhouse gas emissions, global warming and other environmental issues, some misconceptions still prevail and there is a lack of the detailed knowledge required to better understand and judge modern energy technologies and how to improve energy efficiency through behavior change (cf. $[30,31,32]$ ). In regard to the lack of detailed knowledge, two informational strategies will be looked at which are aimed at mitigating knowledge deficiency and reducing the acquisition of imperfect information. These strategies have recently gained momentum in both research and practice, thus acknowledging their promising potential for energy audits and feedback on energy use. Although many of the studies carried out have been only small-scale pilot studies, they do suggest that these strategies deserve some credence.

To begin with, a comparison of industrial audits and household energy audits suggests that the former have a stronger economic dimension related to investment in and deployment of energy efficient technology in respect to cost savings. However, aspects of restructuring processes and tasks aimed at reducing energy consumption are also reflected, and these include behavioral change, though at the level of the organization rather than at the level of the individual as would be the case with household energy audits. It appears that the characteristics of several issues related to knowledge generation, diffusion and innovation, as discussed in the following, are similar. For example, for auditing, the potential to generate knowledge through learning by doing is in most cases ignored. Also, if the knowledge and insight gained during an audit were documented and made available, it would contribute to the collective knowledge on energy auditing. Moreover, through evaluation by others, it would increase in value and make energy auditing more effective and efficient as knowledge spillovers and increasing returns of knowledge supply and utilization, typical dynamics of knowledge societies, get to work. It would also contribute to defining social norms in regard to comparisons with what other social actors, whether individual households or private / public organizations, prefer, and do or do not do anymore, because there would be empirical evidence available. As studies have revealed (cf. [30,32,33,34]), an important aspect in this context is detailed personalized information, as that leads to knowledge that translates into informed decisions on how to achieve goals. The number and complexity of interacting factors is small in household energy audits compared to those in industrial energy audits. Here, therefore, user innovation represents another mostly ignored opportunity for knowledge generation, as discussed elsewhere in this paper. Users need to be given an environment that would permit such activities. Even though the information technology exists and the communication infrastructure is already in place, unfortunately the concerted efforts which are indispensable to make the best use of these are still not in sight.

The situation in the case of feedback on energy use in households seems to be even less favorable, as the differences between what we know and what is actually done in practice in public programs and policies seem considerable, and they are widening further. For example, all empirical studies on energy consumption feedback consistently showed that, to be both effective and efficient, information needs to be presented clearly and regularly, in a tailored manner, giving appliance-specific breakdowns (for an overview of recent work see $[35,36]$ ). This requires an energy infrastructure with smart meters and interactive inhouse displays. Most industrialized nations, however, have smart meter roll-out programs featuring only a simplified metering technology without any appropriate feedback. 
They provide sufficient information for energy utilities to improve their demand-side management and thus their own economic interests, but they deprive the household of almost any chance to become an active part in the process. They are also in conflict with several criteria related to cultural values, such as freedom of choice and privacy. Perhaps, therefore, it is not so surprising that they are struggling with public acceptance. Not only do they oppose social and cultural norms, but they also act as obstacles to technology deployment. Another aspect of this scenario is that, up until now, opportunities have been ignored to involve users on a larger scale as active sources contributing to user innovation. They could help not only to customize artifacts such as actual feedback displays (both hardware and software) but also in the provision of the information itself. Thus, they would be able to further identify, shape and improve parameters of the feedback on energy. In this manner knowledge and insight could also be gained on how energy services, lifestyles, energy consumption behavior and their interrelationships are perceived and valued in the eye of the beholder. There are many successful examples of user innovation in information technology where customization of functions and adaptation of parameters defining the interface between technology and society on a local and individual level have brought about knowledge and innovation. Some of these would have been impossible to achieve by relying solely on traditional methods in both academic settings and commercial laboratory-based research and development.

\subsection{The cultural dimension}

Issues attributed in this paper to the cultural dimension within the context of feedback on energy use, in the literature also identified as contextual factors (for example see [35]), are often overlooked and still poorly understood. There are only a few empirical studies and culture-related assessments in this research field. For example, those reported in [37,38] showed that a certain graphical representation using distribution graphs with little houses as marks employed within feedback on household energy consumption proved to be successful in one country (USA), but a complete failure in another country (Norway). Another culture-related issue not yet given much attention relates to feedback contents in respect to comparisons. For example, Western households prefer either comparisons with their own previous results or normative comparisons. However, Asian households, for example in Japan, prefer comparisons with others rather than with their own previous results (cf. [39,40,41]). There are interesting opportunities here, not only to promote further studies, especially cross-cultural ones, but also to observe what techniques are most effective (and ineffective) in different cultural contexts. We need to deepen our knowledge on this issue, and also to broaden it. Recent work reported in [42] demonstrated how creative work from non-technological disciplines integrated with the latest visualization and information technology could overcome culture-related barriers in information presentation, while at the same time providing new forms of innovation.

A successful government campaign was initiated by the Ministry of the Environment some eight years ago in Japan [43]. It was termed Cool Biz and was aimed at behavior change to mitigate greenhouse gas emissions through the reduction of energy consumption in public and private organizations. It attempted to change the dress code to allow for a reduction in energy use related to airconditioning equipment. This could be seen as a socialcultural innovation which aimed at becoming a permanent element of post-modern Japanese culture. Worth noticing is its conceptual approach, taking into account social and cultural knowledge. It focused on body-related cooling and heating, which is in contrast to the Western culture of space cooling and heating. It stressed the importance of a dress code while sustaining the preference for high quality cloth with newly developed designs, cloth colors and cloth fibers to further increase wearer comfort in a hot and humid climate. The campaign made use of the latest information technologies and knowledge of social marketing to increase public awareness at a rapid pace. It supports online shopping and information-pull, while at the same time successfully reducing social barriers by changing social norms regarding what is acceptable in terms of nationwide work wear. It is careful not to oppose fundamental values in the culture in any way. It is also important to note here that this campaign provides a considerable degree of freedom in respect of choice in what to wear, when to join the campaign (which usually lasts from the end of May to September each year), and, indeed, whether to join it at all.

\subsection{The economic dimension}

Let us regard energy not as an end in itself or a basic commodity, but as an input to the generation of a desired energy service, such as space lighting. In this context, energy efficiency can be seen as one of the many characteristics of a technology and of the artifacts employed. In fact, those defining characteristics reflect a part of the embedded knowledge, which relates to the design, manufacture, functionality and intended use. Depending on the background knowledge available and the eye of the beholder, some parts of this knowledge are more obvious than others. 
In the given context, such knowledge relating to energy efficiency could be provided to the economic actors subject to behavior change, i.e. to customers seen as prospective buyers of energy efficient goods and equipment. This would provide several opportunities, still neglected, to overcome underinvestment in energy efficiency. Such underinvestment is considered in the economics literature on energy efficiency, which is obviously influenced partly by insights into behavioral economics, and is regarded as a market failure attributable to asymmetric knowledge and information. In practice, this situation is reflected in the struggles of the markets and in policy as well as program instruments designed to overcome the knowledge imbalance among manufacturers, sellers, and customers. Information provision in respect to energy efficiency can contribute to improving this situation, if designed and implemented according to what is scientifically known (see discussion in [44]). This can be achieved through programs such as labeling, which are currently either in operation or planned for introduction in over 70 countries worldwide. However, more advanced approaches are required to match increasing requirements. Information about the differences in future operating costs between less-efficient and moreefficient products and appliances needs to be provided to encourage private economic decisions to invest. This type of information can steer customers in a more sustainable direction, but it needs to be designed to answer the questions that are important from the viewpoint of those customers, so the information should not be limited to details of cost-minimization in future technology employment. Novel methods of organizing and implementing the provision of information are required, and these must also take into account the costs of doing so (see also discussions on transaction and search costs in $[45,46])$.

This could be approached by offering products together with novel information provision, in the form of interactive information systems, which should draw on advanced information technology, its infrastructure and characteristics of the knowledge society and its economics. Information from different knowledge and expertise areas could be compiled, integrated and provided in addition to the usual product information. This information could include customer service, marketing, service and maintenance engineering for a range of economic actors such as manufacturers, suppliers, and sellers, all of whom would realize that the economic potential, and therefore the value of knowledge, increases when all can agree to share it. If integrated with information networks and online shopping, information-pull would surely promote its diffusion, while knowledge would be generated through learning-by-using from adopters of energy efficient technology. Knowledge would be appreciated as a positive externality and used to further extend and improve information transfer and provision in the sense outlined, and the chances are that neither asymmetric knowledge and information nor the actual transaction and search costs which are mentioned by economists as obstacles, would remain as market barriers in the sense they are seen now. Of course, to make all this happen, it requires concerted efforts and goal setting with a priority on net benefits. Sustainability and the well-being of society need to be put before the short-term benefits of any actor, either organizational or individual.

\section{Conclusion}

This study has been undertaken within the context of sustainable energy technology issues related to deficiency and uncertainty in knowledge and information. The issues examined have included knowledge deficit, asymmetric information and knowledge, transaction and search costs for information and knowledge (in the literature identified as various factors and determinants pertaining to obstacles within public awareness), social acceptance, market failures impeding the deployment and diffusion of renewable energy technology and difficulties in steering the behavior of social and economic actors towards changes in respect to sustainable energy use. These issues were regarded as nontechnical imperatives actually reflecting operational requirements in regard to the socio-cultural and economic environment.

Using such an approach in combination with the viewpoint taken in this study has provided the momentum and synergy to transform what was initially considered a hurdle into an opportunity to aid problem solving, by seeing it as an input contributing to the formulation of a solution. Several promising results have been obtained relating to various types of innovation, knowledge generation through various modes of learning, and opportunities for knowledge diffusion. This has been achieved by relating troubling issues as outlined to the characteristics of rising knowledge societies and the new dynamics of knowledge economies.

Never in the course of human history has knowledge been as advanced and as widely available as it is now. Even so, it seems that our requirements for information and knowledge and our ability to derive insight and understanding from them in order to put ever more complex and ambitious socio-technological systems to work while maintaining sustainability and balance in respect to both the built and the natural environment, constantly 
outrun by several orders of magnitude our ability to generate that information and knowledge.

\section{References}

[1] Gibbons M, Limoges C, Nowotny H, Schwartzman S, Scott P, Trow M. The New Production of Knowledge: The Dynamics of Science and Research in Contemporary Societies. $10^{\text {th }} \mathrm{ed}$. Thousand Oaks: Sage; 2010.

[2] Castells M. The Rise of the Network Society. $2^{\text {nd }}$ ed. Oxford: Wiley-Blackwell; 2010.

[3] Foray D. Economics of Knowledge. Cambridge: MIT Press; 2004.

[4] Fleck J. Learning by Trying: The Implementation of Configurational Technology. Research Policy 1994; 23(6): 637652.

[5] Dougherty D. Organizing Practices in Services: Capturing Practice-Based Knowledge for Innovation. Strategic Organization 2004; 2(1): 35-64.

[6] Kuhn TS. The Structure of Scientific Revolutions. Chicago: University of Chicago Press; 1970.

[7] Clark J, McLoughlin I, Rose H, King R. The Process of Technological Change: New Technology and Social Choice in the Workplace. Cambridge: Cambridge University Press; 1988.

[8] Fleck J. Innofusion or Diffusation? The Nature of Technological Development in Robotics. Edinburgh: Edinburgh University PICT Working Papers No. 7; 1988.

[9] Devine-Wright P. Rethinking NIMBYism: The Role of Place Attachment and Place Identity in Explaining Place-Protective Action. Journal of Community and Applied Social Science 2009; 19(6): 426-441.

[10] Pasqualetti MJ. Morality, Space and the Power of Wind-Energy Landscapes. Geographical Review 2001; 90(3): 381-394.

[11] Swafford J, Slattery M. Public Attitudes of Wind Energy in Texas: Local Communities in Close Proximity to Wind Farms and Their Effects on Decision-Making, Energy Policy 2010; 38(5): 2508-2519.

[12] Devlin E. Factors Affecting Public Acceptance of Wind Turbines in Sweden. Renewable Energy and Wind Power 2005; 29(6): 503-511.

[13] Walker G, Devine-Wright P. Community Renewable Energy: What Should it Mean?. Energy Policy 2008; 36(2): 497-50o.

[14] Hippel E v. Democratizing Innovation: The Evolving Phenomenon of User Innovation. In: Kahin B, Foray D, editors. Advancing Knowledge and the Knowledge Economy. Boston: MIT Press; 2006. pp. 238-255.

[15] Sjöberg L. Attitudes Toward Technology and Risk: Going Beyond What is Immediately Given. Policy Science 2002; 35(4): 379-40o.

[16] Rohrmann B, Renn O. Risk Perception Research -An Introduction. In: Renn O, Rohrmann B, editors. Cross-Cultural Risk Perception: A Survey of Empirical Studies. Dordrecht: Kluwer Academic Publishers; 200o. pp. 11-54.

[17] Scheer D, Konrad W, Scheel O. Public Evaluation of Electricity Technology and Future Low-Carbon Portfolios in Germany and the USA. Energy, Sustainability and Society 2013; 3(8): 1-13.

[18] Wolsink M, Breukers S. Contrasting the Core Beliefs Regarding the Effective Implementation of Wind Power: An International Study of Stakeholder Perspectives. Journal of Environmental Planning and Management 2010; 53(5): 535-558.
[19] Wüstenhagen R, Wolsink M, Bürer MJ. Social Acceptance of Renewable Energy Innovation: An Introduction to the Concept. Energy Policy 2007; 35(5): 2683-2691.

[20]Webb J. The Mismanagement of Innovation. Sociology 1992; 26(3): 471-492.

[21] Jaffe AB, Newell RG, Stavins RN. A Tale of Two Market Failures: Technology and Environmental Policy. Ecological Economics 2005; 54(2-3): 164-174.

[22] Nemet GF. Demand-Pull, Technology-Push, and GovernmentLed Incentives for Non-Incremental Technical Change. Research Policy 2009; 38(5): 700-709.

[23] REN21. 2011. Renewables 2011: Global Status Report. Paris: Renewable Energy Policy Network for the 21st Century; 2011.

[24]Couture T, Gagnon Y. An Analysis of Feed-In Tariff Remuneration Models: Implications for Renewable Energy Investment. Energy Policy 2010; 38(2): 955-965.

[25] Menanteau P, Finon D, Lamy ML. Prices Versus Quantities: Choosing Policies for Promoting the Development of Renewable Energy. Energy Policy 2003; 31(8): 799-812.

[26]Frondel M, Schmidt CM, Vance C. Germany's Solar Cell Promotion: Dark Clouds on the Horizon. Energy Policy 2008; 36(11): 4198-4204.

[27] Otto HE. Post-Modern Engineering Science: Current Trends and Shortcomings of IT use in Knowledge Generation. Proceedings of International Symposium on Environmentally Conscious Design and Inverse Manufacturing, Kyoto, Japan 2011; Paper-No.: B2-01.

[28]David PA, Foray D. Economic Fundaments of the Knowledge Society. Policy Futures in Education 2003; 1(1): 20-49.

[29] Otto HE. Deficiencies in Current Energy Policymaking in the Rise of Knowledge Economies. Journal of the Japan Institute of Energy. to appear.

[30]Abrahamse W, Steg L, Vlek C, Rothengatter JA. The Effect of Tailored Information, Goal Setting and Tailored Feedback on Household Energy Use, Energy-Related Behaviors and Behavioral Antecedents. Journal of Environmental Psychology 2007; $27(4): 265-276$.

[31] Bord RJ, O'Connor RE, Fischer A. In What Sense Does the Public Need to Understand Global Climate Change?. Public Understanding of Science 2000; 9(3): 205-218.

[32] Anderson ST, Newell RG. Information Programs for Technology Adoption: The Case of Energy-Efficiency Audits. Resource and Energy Economics 2004; 26(1): 27-50.

[33] Desmedt J. Vekemans G, Maes, D. Ensuring Effectiveness of Information to Influence Household Behaviour. Journal of Cleaner Production 2009; 17(4): 455-462.

[34] Gruber E, Fleiter T, Mai M, Frahm, BJ. Efficiency of an Energy Audit Programme for SMEs in Germany - Results of an Evaluation Study. Summer Study Proceedings of European Council for an Energy Efficient Economy, Belambra Presqu'île de Giens, France 2011; pp. 663-674.

[35] Abrahamse W, Steg L, Vlek C, Rothengatter JA. A Review of Intervention Studies Aimed at Household Energy Conservation. Journal of Environmental Psychology 2005; 25(3): 273-291.

[36] Fischer C. Feedback on Household Electricity Consumption: A Tool for Saving Energy?. Energy Efficiency 2008; 1(1): 79-104.

[37] Egan C. Graphical Displays and Comparative Energy Information: What Do People Understand and Prefer?. Summer Study Proceedings of European Council for an Energy Efficient Economy, Paris, France 1999; Paper-No.: 2-12. 
[38] Wilwhite H, Høivik A, Olsen JG. Advances in the Use of Consumption Feedback Information in Energy Billing: The Experiences of a Norwegian Energy Utility. Summer Study Proceedings of European Council for an Energy Efficient Economy, Paris, France 1999; Paper-No.: 3-2.

[39] Ueno T, Inada R., Seaki O, Tsuji K. Effectiveness of Displaying Energy Consumption Data in Residential Houses. Analysis on How the Residents Respond. Summer Study Proceedings of European Council for an Energy Efficient Economy, Mandelieu La Napoule, France 2005; pp. 811-822.

[40]IEADSM. Task XI: Time of Use Pricing and Energy Use for Demand Management Delivery: Subtask 1 - Smaller Customer Energy Saving by End Use Monitoring and Feedback. IEA DSM Report. Chester: International Energy Agency Demand Side Management Programme / EA Technology; 2005.

[41] Sernhed K, Pyrko J, Abaravicius J. Bill Me This Way! - Customer Preferences Regarding Electricity Bills in Sweden. Summer Study Proceedings of European Council for an Energy Efficient Economy, Saint-Raphaël, France 2003; pp. 1147-1150.

[42] Holmes TG. Eco-Visualization: Combining Art and Technology to Reduce Energy Consumption. Proceedings of ACM SIGCHI Conference on Creativity and Cognition, Washington DC, USA 2007; pp. 153-162.

[43] MOE. Result of "Cool Biz" Campaign. Tokyo: Government of Japan, Ministry of the Environment Press Release October 28; 2005.

[44]Egan C, Waide P. A Multi-Country Comparative Evaluation of Labelling Research. Summer Study Proceedings of European Council for an Energy Efficient Economy, Mandelieu La Napoule, France 2005; pp. 811-822.

[45] Howarth R, Andersson B. Market Barriers to Energy Efficiency. Energy Efficiency 1993; 15(4): 262-272.

[46]Wilson, CM. Market Frictions: A Unified Model of Search Costs and Switching Costs. European Economic Review 2012; 56(6): 1070-1086. 